\title{
Further Mycotoxins and Microbial Metabolites
}

Aquatic food (literature ${ }^{536}$ ) contained further mycotoxins/ microbial metabolites:

Tentoxin

Chicken gizzard (literature ${ }^{326}$ ) contained further mycotoxins/ microbial metabolites:

a-Zearalanol, ß-zearalanol, and a-zearalenol

Chicken heart (literature ${ }^{326}$ ) contained further mycotoxins/ microbial metabolites:

a-Zearalanol, ß-zearalanol, and a-zearalenol

Chicken liver (literature ${ }^{326}$ ) contained further mycotoxins/ microbial metabolites:

a-Zearalanol, B-zearalanol, and a-zearalenol

Dairy products (literature ${ }^{457}$ ) contained further mycotoxins/ microbial metabolites:

Ochratoxin-a

Eggs (literature ${ }^{326}$ ) contained further mycotoxins/microbial metabolites:

a-Zearalanol, ß-zearalanol, a-zearalenol, and ß-zearalenol

Eggs (literature ${ }^{535}$ ) contained further mycotoxins/microbial metabolites:

ß-Zearalenol

Eggs (literature ${ }^{536}$ ) contained further mycotoxins/microbial metabolites:

Tentoxin

Milk (cow milk) (literature ${ }^{231}$ ) contained further mycotoxins/ microbial metabolites:

Aflatoxicol
Milk (cow milk) (literature ${ }^{456}$ ) contained further mycotoxins/ microbial metabolites:

a-Zearalenol

Milk (human breast milk) (literature ${ }^{315}$ ) contained further mycotoxins/microbial metabolites:

Ochratoxin-a

Milk (human breast milk) (literature ${ }^{320}$ ) contained further mycotoxins/microbial metabolites:

Aflatoxicol

Milk (human breast milk) (literature ${ }^{321}$ ) contained further mycotoxins/microbial metabolites:

Aflatoxicol

Milk (human breast milk) (literature ${ }^{472}$ ) contained further mycotoxins/microbial metabolites:

Aflatoxicol

Milk (human breast milk) (literature $\mathrm{e}^{513}$ ) contained further mycotoxins/microbial metabolites:

a-Zearalenol and B-zearalenol

Milk (infant formula) (literature ${ }^{164}$ ) contained further mycotoxins/microbial metabolites:

a-Zearalenol and B-zearalenol

Milk powder (literature ${ }^{456}$ ) contained further mycotoxins/ microbial metabolites:

a-Zearalenol

For incidence and concentration as well as possible cocontamination of these compounds see the corresponding article. 